\title{
On Improving Communication Robustness in PLC Systems for More Reliable Smart Grid Applications
}

\author{
Khaled M. Rabie, Student Member, IEEE, Emad Alsusa, Senior Member, IEEE
}

\begin{abstract}
Power-line communication (PLC) has been the main enabler for modernizing the aging electrical power grid. As such, PLC systems have been the subject of intensive research in the community. One of the major aspects of PLC is the link interface, for which orthogonal frequency-division multiplexing (OFDM) has been widely adopted. In this paper we propose the application of orthogonal poly-phase based multi-carrier code division multiple access (OPP-MCCDMA) due to its inherent better flexibility and signal-envelope properties which can be utilized to further enhance the reliability of PLC signals. The proposed OPP-MC-CDMA system is implemented with a minimum mean square error equalizer and nonlinear preprocessing to overcome the effects of bursty noise and multipath frequency-selective fading commonly experienced in PLC channels. We study the performance of this system in terms of the output signal-to-noise ratio (SNR) and symbol error rate with various constellation sizes of OPP codes under different noise scenarios and nonlinear processor's thresholds. For comparison-sake, the performance of the OFDM scheme is included. The results reveal that the proposed approach always provides superior performance over the OFDM one with a maximum output SNR gain of up to 5.25 dB. It is also shown that the performance of the OPP-MC-CDMA technique improves when increasing the constellation size of the OPP codes which consequently enhances the reliability of PLC.
\end{abstract}

Index Terms-Blanking, clipping, convolutional codes, impulsive noise, multi-carrier code division multiple access (MC-CDMA), multipath fading channel, orthogonal poly-phase (OPP) codes, power-line communications (PLC), smart grid (SG).

\section{INTRODUCTION}

$\mathbf{N}$ OWADAYS everything is expected to conform to the concept of smart environments, not least of which the electric grid. The considerable domestic and industrial reliance on electricity has significantly strained such a limited resource and this, consequently, has driven utility companies to realize the urgency to modernize the existing and aging grid in order to efficiently cope with the modern society requirements. Thanks to advances in communications many smart grid (SG) services and functionalities have been made possible such as remote power grid configuration, dynamic pricing, advanced metering and load control [1], [2]. It is broadly believed that, for more efficient realization of SG, a heterogeneous set of networks should be adopted such as Wi$\mathrm{Fi}$, coaxial-cables, fiber optics, power-line networks etc, since no single technology can be a perfect solution for all scenarios [2]. However, the fact that power-line communication (PLC) technology incurs no additional installation costs makes it more attractive to SG developers than the other alternatives. Not only that, but also because PLC is a through-grid technology, it reduces the reliance of utility companies on third party connectivity, which of course will result in a cost reduction, and eliminate security and privacy issues [3]. PLC is, generally, divided into two main categories, namely, narrow-band PLC (NB-PLC) and broad-band PLC (BBPLC) both of which can offer solutions for different smart grid

The authors are with the School of Electrical and Electronic Engineering, the University of Manchester, Manchester, M13 9PL, UK. (emails: khaled.rabie@manchester.ac.uk; e.alsusa@manchester.ac.uk).

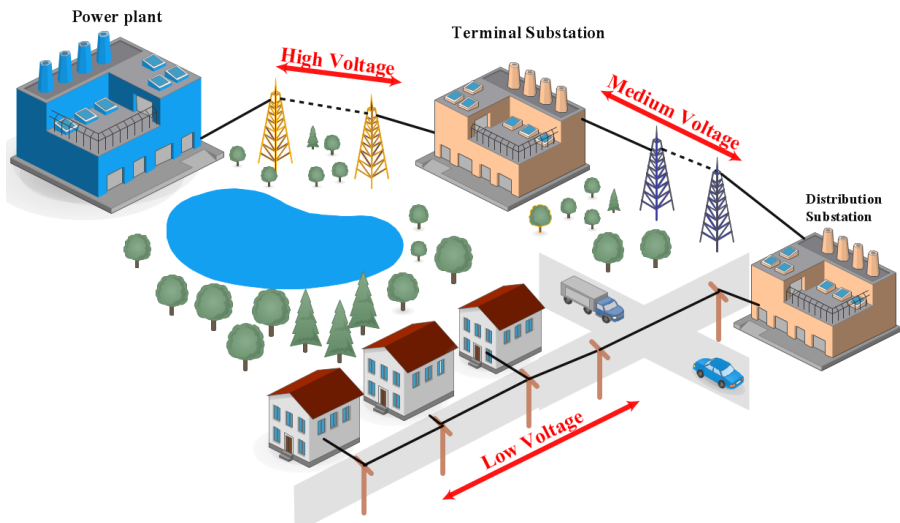

Figure 1: Schematic diagram of power-line communication systems.

scenarios and applications such as advanced metering infrastructure (AMI), home automation etc [4]-[8]. NB-PLC mainly exploits the low and medium voltage distribution network whereas BB-PLC utilizes the low voltage distribution network as illustrated in Fig. 1. It should be mentioned that the focus of this paper will be mainly on BB-PLC.

It is well acknowledged that power-line channels are a very harsh and noisy transmission medium suffering tremendously from frequency selectivity, time-varying topology issues, electromagnetic interference and noise. Many studies have concluded that noise is the most crucial element influencing the reliability of PLC-based SG applications which is, in general, classified into background noise (BN) and impulsive noise (IN) [9]-[11]. It is also found that IN can have very high amplitudes with durations frequently exceeding the signal symbol length which can dramatically worsen quality of service [9], [12]. In order to emulate both types of noise and obtain reasonable predictions of BB-PLC system performance, Middleton class-A noise model, [13], [14], is commonly used by PLC researchers [15], and likewise, it will be adopted in this paper. To reduce the adverse effect of IN, blanking, clipping, or a combination of both (hybrid), is usually applied at the front-end of the receiver to zero or/and clip the incoming signal when it exceeds a certain threshold value [16], [17].

However, the performance of such techniques is highly affected by the type of link interface used. Orthogonal frequency division multiplexing (OFDM) is a widely adopted interface for PLC systems due to its ability to convert a frequency-selective channel to a set of flat fading channels. However, it has been shown that IN cancellation is less effective due to its high signal-envelop fluctuations. As such, and for more efficient SG applications, in this paper we investigate the performance of multi-carrier code-division multiple access (MC-CDMA), [18]-[20], with blanking, clipping and adaptive hybrid over multipath PLC channels which provides granularity in both time and frequency domains, hence allowing a high degree of flexibility. While both MC-CDMA and conventional 
Transmitter

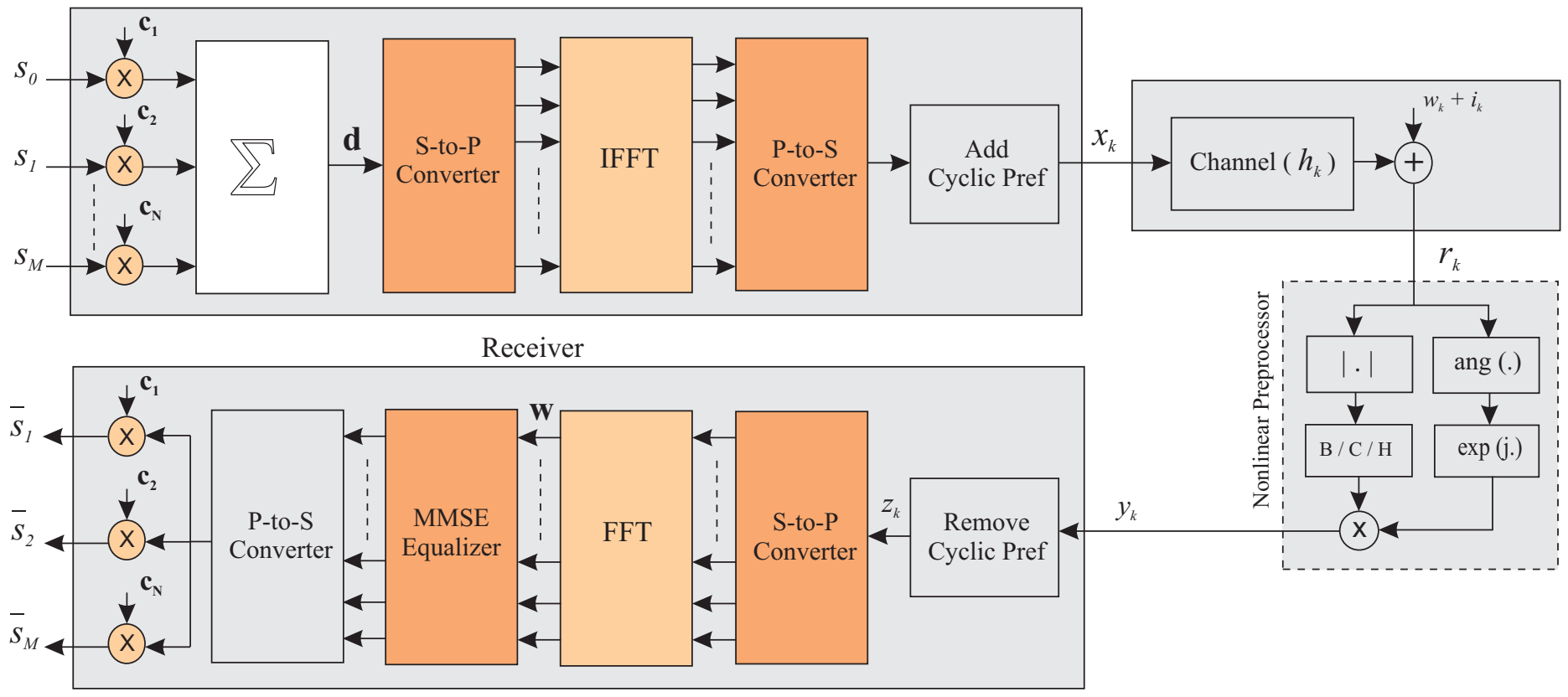

Figure 2: Block diagram of the OPP-MC-CDMA system with a nonlinear preprocessing device at the receiver.

OFDM transmit symbols in parallel, they differ in the sense that the latter transmits different symbols over the available sub-carriers whereas in the former each symbol is spread over the entire number of sub-carriers, hence both systems still provide equal data rates. Although no specific SG scenario is considered here, the proposed system is generally applicable to any SG application utilizing the power-line network; for example, it could be used for smart meters and home automation applications. The motivations for using CDMA for PLC includes, firstly, its flexibility as a multiple access system compared to other PLC OFDM-based standards which rely on the carrier sense multiple access-collision avoidance (CSMA-CA) algorithm [21]-[24]. This can, consequently, reduce system complexity and delay considerably. Secondly, its resilience to IN and its lower peak-to-average power ratio (PAPR) features, compared to OFDM, for certain types of sequences make CDMA a very attractive alternative. It should be indicated that several studies have already investigated the performance of these systems over wire-line channels such as digital subscriber line (DSL) and powerlines; in particular, the authors of [25]-[27] considered the problem of resource allocation and synchronization.

To realize MC-CDMA, [28], many classes of spreading sequences can be utilized such as Pseudonoise (PN), Gold, Kasami, Walsh-Hadamard (WH) and orthogonal poly-phase (OPP) sequences [29], [30]. In this paper, however, we consider MCCDMA with OPP sequences which are the non-binary extension of the binary WH sequences. The rationale for selecting these codes includes: firstly, their robustness against timing misalignment which can significantly degrade the performance of the binary WH codes. Secondly, OPP codes have similar cross-correlation properties as the binary $\mathrm{WH}$ and, unlike the latter, OPP codes are not limited by the sequence length hence can accommodate much more users/data. Thirdly, but most importantly, such codes tend to have lower PAPR properties as the constellation size of these codes is increased. The utmost importance of this property resides in the fact that minimizing the PAPR of the transmitted signal will ultimately flatten the envelop of the signal which will allow more efficient blanking and/or clipping of IN at the receiver and hence improve the overall performance. It should also be mentioned that a minimum mean square error (MMSE) equalizer is employed at the receiver to compensate for the inter-symbol interference (ISI) caused by the frequency-selective channel without increasing the noise power.

The contribution of this paper is threefold. First the PAPR performance of the OPP-MC-CDMA approach is studied for various constellation sizes of OPP codes under full-loading and half-loading system scenarios to establish the critical relationship between the system loading, OPP code constellation size and PAPR of the transmitted signal. This is important as it was shown [31] that signals with lower PAPR are more resilient to bursty noise. The second contribution resides in evaluating the signal-to-noise ratio (SNR) value at the output of the most three popular nonlinear preprocessors for reducing the impact of bursty noise in multipath fading. Finally, the problem of blanking and clipping thresholds optimization is addressed in different noise environments and the corresponding maximum achievable output SNR and minimum SER performances are evaluated. Although this paper mainly focuses on the analysis of OPP-MC-CDMA in the context of improving the reliability of SG applications, for the purpose of comparison, OFDM-based schemes are also included throughout our investigations. The results indicate that OPP-MCCDMA with blanking and clipping can offer up to $5.25 \mathrm{~dB}$ and $2.25 \mathrm{~dB}$ improvement in the output SNR relative to the OFDMbased schemes, respectively. It is also shown that the proposed system is always able to considerably enhance performance even when the constellation size of OPP codes is relatively small and this enhancement increases as the number of OPP codes phases is increased.

This paper is organized as follows. In Section II the system model is described. Section III discusses the PAPR performance of the proposed system for various OPP codes phases. Section IV is dedicated to study the output SNR of the proposed system. In Section $\mathrm{V}$ the maximum achievable output SNR and the minimum SER 
performances that correspond to the optimal blanking and clipping thresholds are examined for various noise scenarios. The impact of Middleton class-A impulsive index on the overall performance of the optimized system is outlined in Section VI. Section VII presents a bit error rate (BER) performance comparison between the proposed MC-CDMA and the coded OFDM systems. Finally, Section VIII gives a summary and some concluding remarks.

\section{SySTEM MOdeL}

The system model under consideration is illustrated in Fig. 2. First, the 16-QAM symbol $\left(s_{m}\right)$ of each user is spread using the user-specific code $\mathbf{c}_{m}=\left[c_{m}^{(0)}, c_{m}^{(1)}, \ldots, c_{m}^{(N-1)}\right]$, where $m=[1,2, \ldots, M], M$ is the total number of users and $N$ denotes the code length. It should be stated here that, throughout this work unless explicitly stated otherwise, we use a fully loaded system (i.e. $M=64$ users). After that, the spread signals are multiplexed to produce $\mathbf{d}=\left[d_{0}, d_{1}, \ldots, d_{N-1}\right]$ which is then passed through a serial-to-parallel (S/P) convertor. The S/P output is fed to an inverse fast Fourier transform (IFFT), the size of which is equal to the code length $(N)$, then applied to a parallel-to-serial $(\mathrm{P} / \mathrm{S})$ convertor and a cyclic prefix is added before transmission. The transmitted signal for one MC-CDMA block is expressed mathematically as

$$
x(t)=\sum_{i=0}^{N-1} \sum_{m=0}^{M-1} s_{m} c_{m}^{(i)} e^{\left(j \frac{2 \pi i t}{T_{s}}\right)}
$$

where $T_{s}$ is the MC-CDMA symbol duration. Using this definition in (1), the PAPR of the MC-CDMA transmitted signal is given by

$$
\text { PAPR }=10 \log _{10}\left(\frac{\max \left|x_{k}\right|^{2}}{\mathbb{E}\left|x_{k}\right|^{2}}\right)
$$

where $\mathbb{E}$ is the expectation operator and $x_{k}=x\left(k T_{s} / N L\right)$. Oversampling of 4 is implemented in this study as such oversampling rate is shown in the literature to provide accurate estimates of the PAPR [32]. After the signal is passed through the multipath PLC channel, the received signal can be written as

$$
r_{k}=x_{k} * h_{k}+n_{k}, \quad k=0,1, \ldots, L N-1
$$

while ' $*$ ' denotes convolution operation, $h_{k}$ is the impulse response of multipath fading channel and $n_{k}$ is the total noise component. In this study we adopt a standard Rayleigh multipath fading channel for both OFDM and MC-CDMA systems and assume that the number of channel taps is lower that the cyclic prefix duration to avoid the ISI problem, i.e. orthogonality is maintained by the cyclic prefix. It should also be pointed out that perfect channel estimation is assumed and hence the results will serve as an upper bound for the performance.

As mentioned in the introduction, Middleton class-A noise model is adopted here to emulate the total noise characteristics. This model considers both BN and IN components and has the following probability density function (PDF)

$$
p(z)=\sum_{m=0}^{\infty} \frac{e^{-A} A^{m}}{m !} \cdot \frac{1}{\sqrt{2 \pi \sigma_{m}^{2}}} \exp \left(-\frac{|z|^{2}}{2 \sigma_{m}^{2}}\right)
$$

where

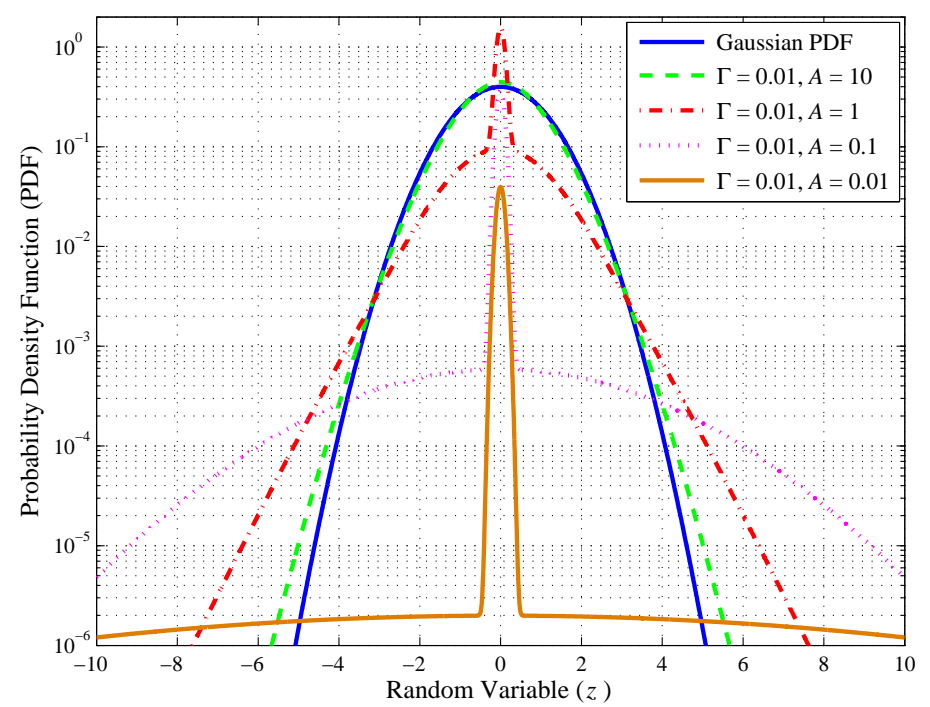

Figure 3: PDFs of Middleton class-A noise for different values of A when $\Gamma=0.01$

$$
\sigma_{m}^{2}=\sigma_{u}^{2}\left(\frac{\frac{m}{A}+\Gamma}{1+\Gamma}\right), \sigma_{u}^{2}=\sigma_{G}^{2}+\sigma_{I}^{2} \text { and } \Gamma=\frac{\sigma_{G}^{2}}{\sigma_{I}^{2}}
$$

while $\sigma_{m}^{2}$ denotes the variance of the $m^{\text {th }}$ considered IN source, $\sigma_{u}^{2}$ is the total noise power, $\sigma_{G}^{2}$ is the Gaussian noise power, $\sigma_{I}^{2}$ is the impulsive (non-Gaussian) noise power, $A=E\{m\}=$ $\sum_{m=0}^{\infty}\left(m e^{-A} A^{m} / m !\right)$ representing the the average number of impulsive sources simultaneously active and is referred to as impulsive index and $\Gamma$ denotes the ratio between the average power of the Gaussian component and the impulsive component; $\Gamma$ is also referred to as the Gaussian-to-impulsive ratio (GIR). It should be mentioned here that there are other noise modeling schemes reported in the literature most of which are empirically-based [33][35]. Having a closer look at (4), however, it can easily be noticed that the PDF of Middleton class-A model (4) is, basically, a sum of weighted normal distributions. It is clear that the three parameters $A, \Gamma$ and $\sigma_{u}^{2}$ specify the statistical characteristic of this model and that when $A$ is large IN will become continuous and, therefore, Middleton class-A noise becomes more likely as Gaussian noise; while conversely, low values of $A$ means rare and highly structured IN. To visualize this, numerical results, obtained from (4) for $(m=0 \rightarrow 100)$, are illustrated in Fig. 3 for various values of $A$ when $\Gamma=0.01$ along with the Gaussian PDF. It is apparent from this figure that when $A$ is large $(A=10)$ the distribution of Middleton class-A noise is very similar to Gaussian distribution but becomes more impulsive as $A$ is reduced.

In order to diminish the deleterious impact of IN, the received signal $\left(r_{k}\right)$ is passed through one of the following nonlinear preprocessors

- Blanking

$$
y_{k}=\left\{\begin{array}{ll}
r_{k}, & \left|r_{k}\right| \leq T_{b} \\
0, & \left|r_{k}\right|>T_{b}
\end{array} \quad k=0,1, \ldots, L N-1\right.
$$

where $T_{b}$ is the blanking threshold. 
- Clipping

$$
y_{k}=\left\{\begin{array}{ll}
r_{k}, & \left|r_{k}\right| \leq T_{c} \\
T_{c} e^{j \arg \left(r_{k}\right)}, & \left|r_{k}\right|>T_{c}
\end{array} \quad k=0,1, \ldots, L N-1\right.
$$

where $T_{c}$ is the clipping threshold.

- Adaptive Hybrid

$$
y_{k}= \begin{cases}r_{k}, & \left|r_{k}\right| \leq T_{a h} \\ T_{a h} e^{j \arg \left(r_{k}\right)}, & T_{a h}<\left|r_{k}\right| \leq \alpha T_{a h} \\ 0, & \left|r_{k}\right|>\alpha T_{a h}\end{cases}
$$

where $r_{k}$ and $y_{k}$ are the input and the output of the nonlinear devices, respectively; whereas $\alpha$ is a scaling factor $(\alpha>1)$ which defines the ratio between the blanking and clipping thresholds. Above all, the adaptive hybrid scheme was found to establish the lower bound performance of the nonlinear preprocessing-based method since it optimizes not only the threshold but also the scaling factor [36]. It is important to emphasize that all the nonlinear devices only modify the amplitude of the received signal while leaving its phase unchanged.

After the nonlinear device, the next stage is the $\mathrm{CP}$ removal after which the resultant signal $\left(z_{k}\right)$ is fed to the S/P converter and then to an $N$-point fast Fourier transform (FFT) to produce $\mathbf{W}=\left[W_{0}, W_{1}, \ldots, W_{N-1}\right]$. In order to compensate the channel distortion, equalization is performed using the MMSE equalizer as [37]

$$
W(k)=\frac{H^{*}(k)}{H(k) H^{*}(k)+\mathrm{SNR}_{\mathrm{out}}^{-1}}
$$

where $\mathrm{SNR}_{\text {out }}$ represents the SNR at the output of the nonlinear preprocessor and $H(k)$ is the channel frequency response. After that, the output of the MMSE equalizer is passed through a P/S converter which is then multiplied by the spreading codes to produce estimates for the data symbols of the different users $\left(\bar{s}_{m}\right)$. Finally, 16-QAM demodulation takes place and the SER can be calculated.

\section{PAPR RELATIONSHIP TO OPP CONSTELLATIONS}

In this section we investigate the impact of the number of OPP codes phases $(\phi)$ on the PAPR performance at the transmitter side. Before proceeding with this, however, the complementary cumulative distribution function (CCDF) of the PAPR should be introduced. It is defined as the probability that the PAPR of a data block exceeds a given threshold $\left(\mathrm{PAPR}_{o}\right)$ and is expressed as $\mathrm{CCDF}=1-P\left(\mathrm{PAPR} \leq \mathrm{PAPR}_{o}\right)$. Fig. 4 depicts the PAPR performance of the OPP-MC-CDMA signal against $\phi$ for two loading scenarios: half-loading $(M=32)$ and full-loading $(M=64)$ when $\mathrm{CCDF}=10^{-1}, 10^{-2}$ and $10^{-3}$. The first observation one can see from these results is that the PAPR value is reduced with increasing the number of OPP codes phases. Comparing the two loading scenarios, it is obvious that full-loading has always lower PAPR with respect to half-loading at the same CCDF and $\phi$ values. It is also apparent that the amount of PAPR reduction is more significant in full-loading than that with halfloading. For example, in the former case at $\mathrm{CCDF}=10^{-3}$, a PAPR reduction of about $6 \mathrm{~dB}$ can be achieved when $\phi=64$ relative to the system with $\phi=2$ whereas only about $4.5 \mathrm{~dB}$ is attained from the half-loading scenario at the same CCDF value. This enhancement can be intuitively justified by the increase in

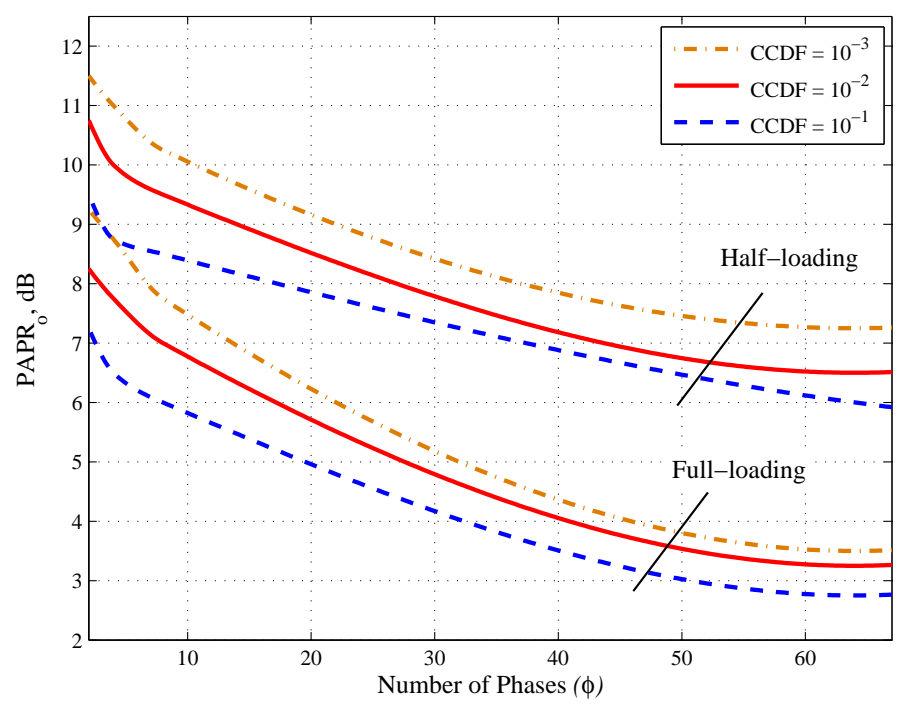

Figure 4: PAPR of OPP-MC-CDMA system versus the constellation size of OPP codes for various CCDF values with full-loading and half-loading.

the phase randomization across the sub-carriers which is related to the minimum-distance decoding of the sequences [38]. This, eventually, leads to more averaging of the transmitted MC-CDMA signal and consequently improves the overall PAPR performance. Furthermore, it is noteworthy to mention that the PAPR tends to level off for very large constellation sizes of OPP codes. Recalling the OFDM-based results found in [31], minimizing the signals' peaks implies that IN will become more easily identifiable at the receiver and, subsequently, will allow more accurate and efficient noise cancellation.

Although in this section we looked into both half-loading and full-loading scenarios, in the rest of this paper, without loss of generality, the focus will be primarily on the latter not only because it relatively has better PAPR properties but also because full loading is a more common configuration in practice.

\section{Preprocessor Threshold Impact on the OUtPut SNR}

In this section we assess the performance of OPP-MC-CDMA with different constellation sizes in terms of SNR at the output of the nonlinear preprocessors. The output SNR is determined as

$$
\mathrm{SNR}_{\text {out }}=\frac{2 K_{o}^{2}}{E_{\text {out }}-2 K_{o}^{2}}
$$

where $K_{o}$ is determined as $K_{o}=(1 / 2) \mathbb{E}\left[\left|y_{k} x_{k}^{*}\right|\right]$ and $E_{\text {out }}=$ $\mathbb{E}\left[\left|y_{k}\right|^{2}\right]$. Our investigations from this section onward, unless stated otherwise, are based on: $M=64$ users, sampling rate is 4 samples per sub-carrier, transmitted signal is normalized as $\sigma_{x}^{2}=(1 / 2) \mathbb{E}\left[\left|x_{k}\right|^{2}\right]=1, \sigma_{u}=0.05, A=0.0025$ and $\Gamma=0.001$ which means that IN is 1000 times, or $30 \mathrm{~dB}$, greater than the BN level. Fig. 5 shows the output SNR of the proposed system versus the blanking/clipping threshold for the three nonlinear preprocessing schemes with different OPP codes phases $\{\phi=2,4,8,64\}$ in a multipath fading channel. In addition, the performance of OFDM-based schemes is also included with and without multipath fading. Note that for fair comparisons same channel is used for 


$$
K_{o}=1-\sum_{m=0}^{\infty} p_{m}\left[e^{-\frac{T^{2}}{2\left(1+\sigma_{m}^{2}\right)}}+\frac{T^{2}}{2\left(1+\sigma_{m}^{2}\right)} e^{-\frac{\alpha^{2} T^{2}}{2\left(1+\sigma_{m}^{2}\right)}}\right]-\sum_{m=0}^{\infty} p_{m} \sqrt{\frac{\pi}{2}} \frac{T}{\sqrt{1+\sigma_{m}^{2}}}\left[Q\left(\frac{T}{\sqrt{1+\sigma_{m}^{2}}}\right)-Q\left(\frac{\alpha T}{\sqrt{1+\sigma_{m}^{2}}}\right)\right]
$$

$$
E_{\text {out }}=2+2 \sum_{m=0}^{\infty} p_{m}\left(\sigma_{m}^{2}-\left(1+\sigma_{m}^{2}\right) e^{-\frac{T^{2}}{2\left(1+\sigma_{m}^{2}\right)}}-\frac{T^{2}}{2} e^{-\frac{\alpha^{2} T^{2}}{2\left(1+\sigma_{m}^{2}\right)}}\right)
$$

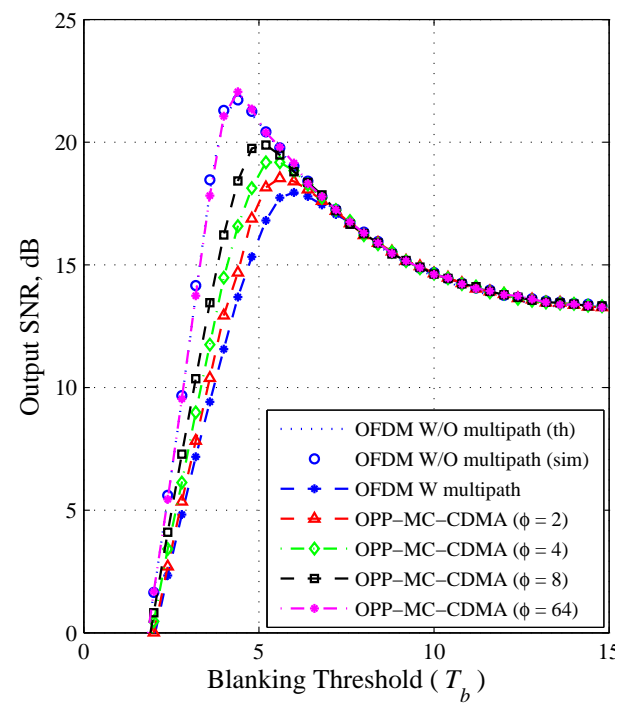

(a) Blanking

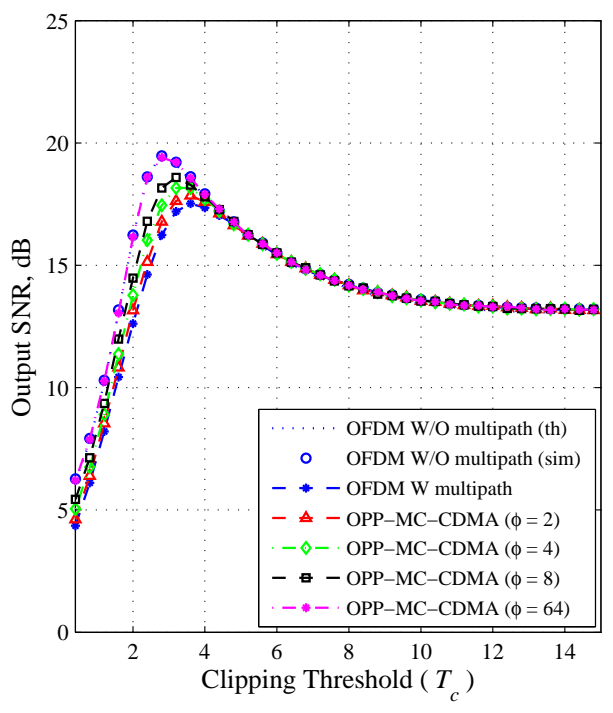

(b) Clipping

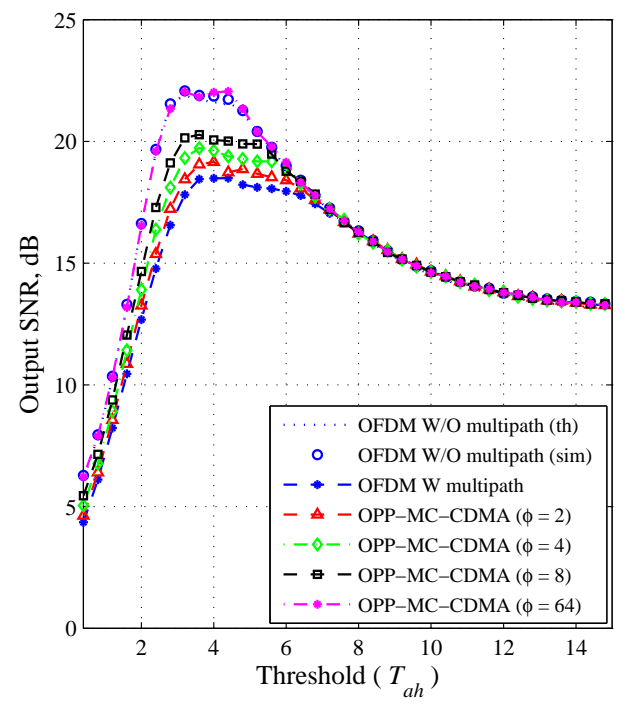

(c) Adaptive Hybrid

Figure 5: Output SNR as a function of the blanking/clipping threshold for OPP-MC-CDMA with various values of $\phi$ and OFDM in the presence of Middleton class-A noise. OPP-MC-CDMA results are obtained for a multipath channel. Noise parameters: $\sigma_{u}=0.05, \Gamma=0.001$ and $A=0.0025$.

both MC-CDMA and OFDM systems. It should be pointed out that the analytical results of the OFDM system W/O multipath for the blanking and clipping techniques are found using (10) with $K_{o}$ and $E_{\text {out }}$ expressions derived in [17]; whereas, for the adaptive hybrid, these parameters found in [36] for the two component mixtureGaussian noise model and are rewritten as in (11) and (12), for the general Middleton class-A noise model.

As a first common observation on the results shown in Figs. $5 \mathrm{a}, 5 \mathrm{~b}$ and $5 \mathrm{c}$, it is clear that the analytical results of OFDM W/O multipath and the simulated ones are in good agreement. As anticipated, it is obvious that, in a multipath channel and irrespective of the nonlinear device utilized, the performance of the OPP-MC-CDMA system always outperforms the OFDM approach even with a small number of phases $($ e.g. $\phi=2)$ and this improvement becomes higher as the code constellation size is increased. Clearly, the proposed system with $(\phi=64)$ can offer output SNR gains of up to $4 \mathrm{~dB}, 2 \mathrm{~dB}$ and $4 \mathrm{~dB}$ relative to the OFDM-based one when blanking, clipping and adaptive hybrid are employed, respectively. Interestingly enough, however, it is evident that when the constellation size of OPP codes is sufficiently large $(\phi=64)$, OPP-MC-CDMA can always achieve exact performance as OFDM W/O multipath. Notably, in both OFDM and OPPMC-CDMA approaches, the adaptive hybrid scheme serves as an upper bound for the performance compared to blanking and clipping cases. Another general trend can be noticed for the three aforementioned nonlinear devices that is when the blanking and/or clipping threshold is very large, i.e. no nonlinear preprocessing is performed (typical receiver), the output SNR approaches $13 \mathrm{~dB}$ in which scenario it can be expressed mathematically as

$$
\operatorname{SNR}_{\text {out }}\left(T_{b, c, a h} \rightarrow \infty\right)=\frac{1}{\sum_{m=0}^{\infty} p_{m} \sigma_{m}^{2}}
$$

which can also be written in terms of Middleton class-A noise parameters as

$$
\operatorname{SNR}_{\text {out }}\left(T_{b, c, a h} \rightarrow \infty\right)=\left(\sigma_{u}^{2} \sum_{m=0}^{\infty} p_{m}\left(\frac{\frac{m}{A}+\Gamma}{1+\Gamma}\right)\right)^{-1}
$$

As a final remark on the results in this section, it is interesting to observe that for every phase value there is always an optimal blanking/clipping threshold that maximizes the output SNR performance. The performance under optimal blanking or/and clipping conditions is investigated next for different noise scenarios.

\section{PERFORMANCE OPTIMIZATION}

In this section extensive computer simulations are conducted to find the optimal blanking/clipping thresholds, the maximum achievable output SNR and the minimum achievable SER performance $^{1}$ for the OPP-MC-CDMA and OFDM systems under various

\footnotetext{
${ }^{1}$ The maximum achievable output SNR and the minimum achievable SER are defined as the output SNR and SER performance that correspond to the optimal blanking/clipping threshold, respectively.
} 


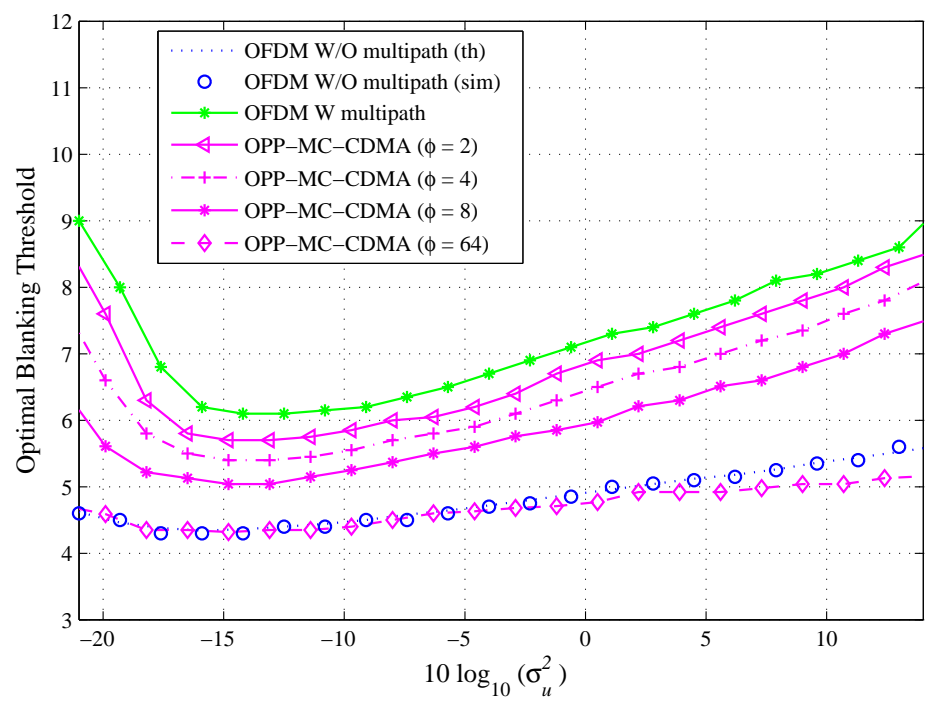

(a) Blanking

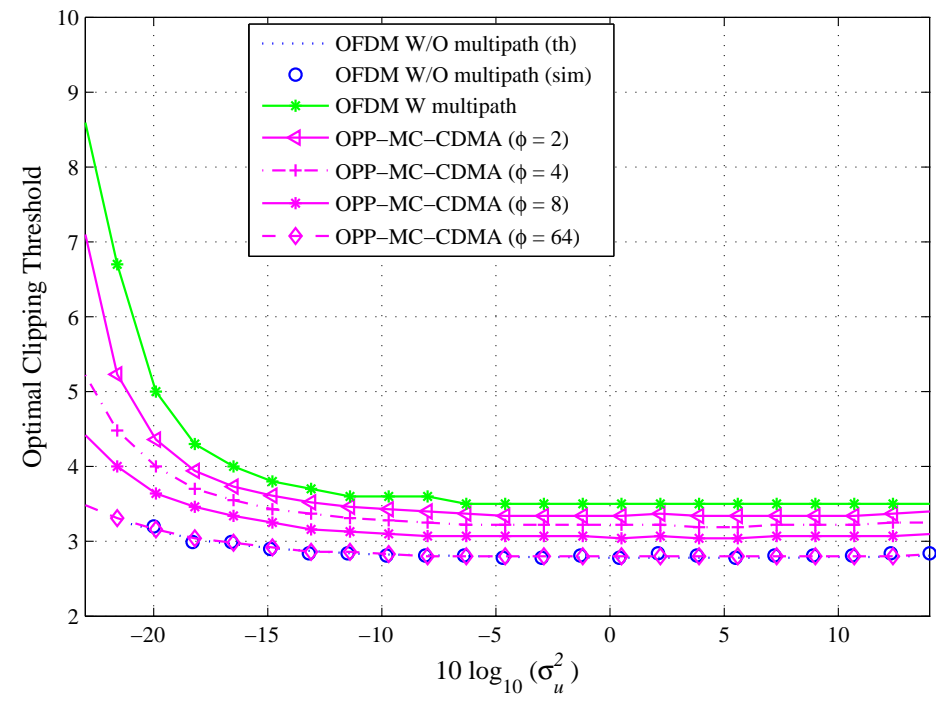

(b) Clipping

Figure 6: The Optimal blanking and clipping thresholds against $10 \log _{10}\left(\sigma_{u}^{2}\right)$ for MC-CDMA with various values of $\phi$ and OFDM systems in a multipath channel with blanking and clipping. Noise parameters: $A=0.0025$ and SGNR $=30 \mathrm{~dB}$.

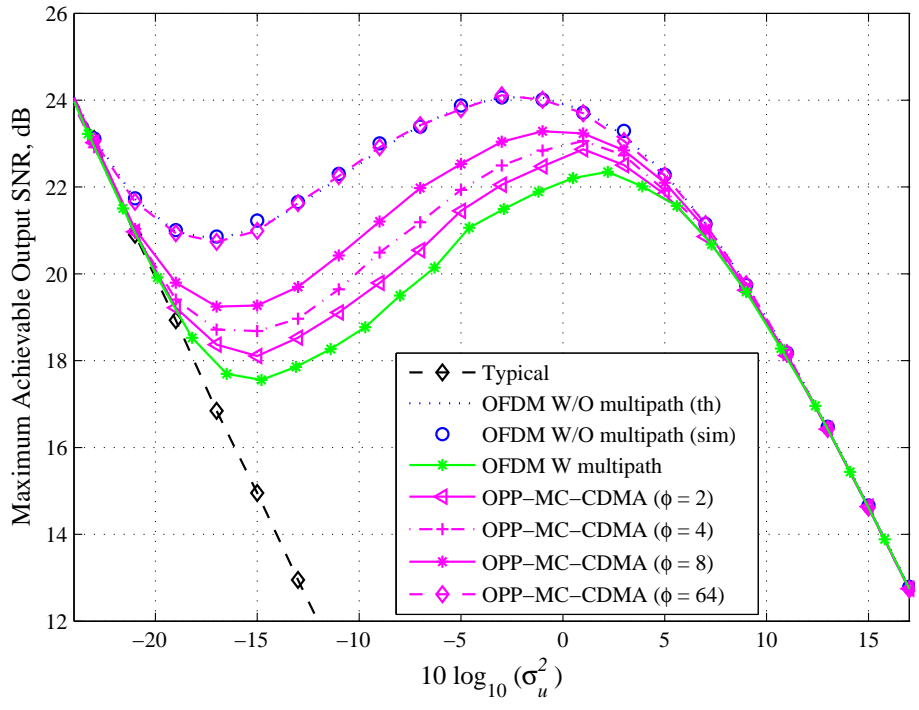

(a) Blanking

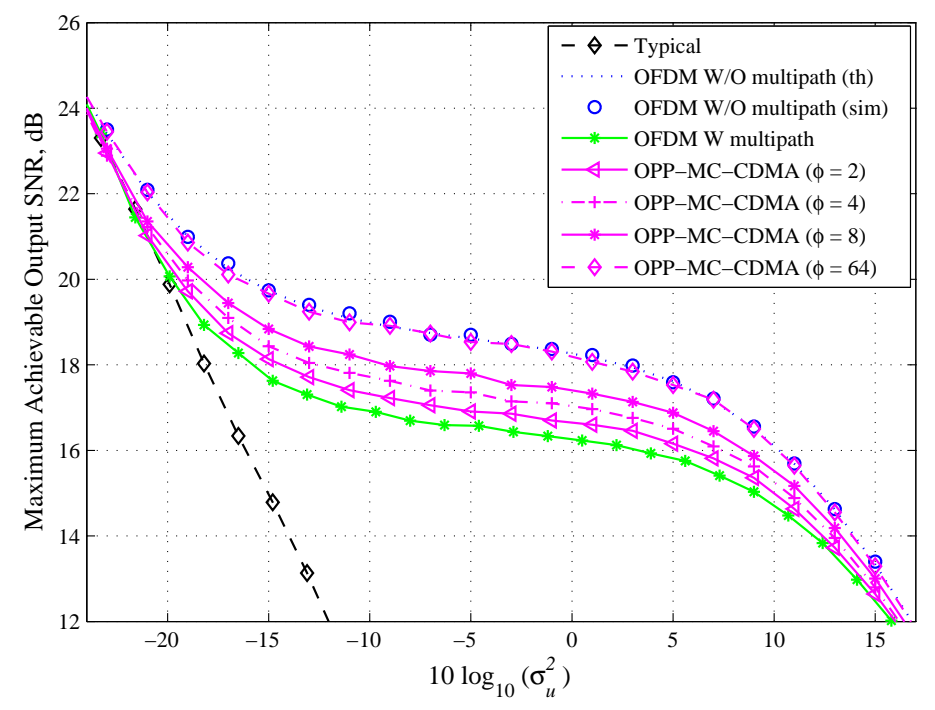

(b) Clipping

Figure 7: Maximum achievable output SNR corresponding to the optimal blanking/clipping threshold versus $10 \log _{10}\left(\sigma_{u}^{2}\right)$ for the MC-CDMA and OFDM systems in a multipath channel for various codes phases with blanking and clipping. Noise parameters: $A=0.0025$ and $\Gamma=0.001$.

noise scenarios. To attain this for the OFDM-based systems W/O multipath, it is more convenient to rewrite (10) as

$$
\left(\mathrm{SNR}_{\text {out }}\right)^{-1}=\frac{E_{\text {out }}}{2 K_{o}^{2}}-1
$$

Clearly, the solution of (15) is difficult to express in closed form and out of the the scope of this paper. Therefore, we will proceed numerically by satisfying the following argument

$$
\min _{T_{b, c}}\left\{\frac{E_{\text {out }}}{K_{o}^{2}}\right\}
$$

It should be mentioned that from this point onward, only blanking and clipping are considered, not only because adaptive hybrid was shown to offer insignificant improvement compared to the other two nonlinear devices, but also because it is more complex to achieve optimal performance as it requires optimizing two parameters $\left(T_{a h}\right.$ and $\left.\alpha\right)$, instead of one. For the OPP-MCCDMA and OFDM with multipath, the optimal thresholds are found by means of simulation by satisfying the following argument

$$
T_{o p t}^{b / c}=\arg \max _{0 \leq T_{b / c}<\infty}\left\{\mathrm{SNR}_{\text {out }}\right\}
$$

Fig 6 depicts some results for the optimal blanking and clipping thresholds versus the noise power, $10 \log _{10}\left(\sigma_{u}^{2}\right)$, for both the MC-CDMA and OFDM systems with and without multipath. It is evident that for both nonlinear preprocessing-based systems the optimal thresholds for MC-CDMA with $\phi=64$ closely match that of the OFDM system W/O multipath. Another common observation in both blanking and clipping schemes is that the 


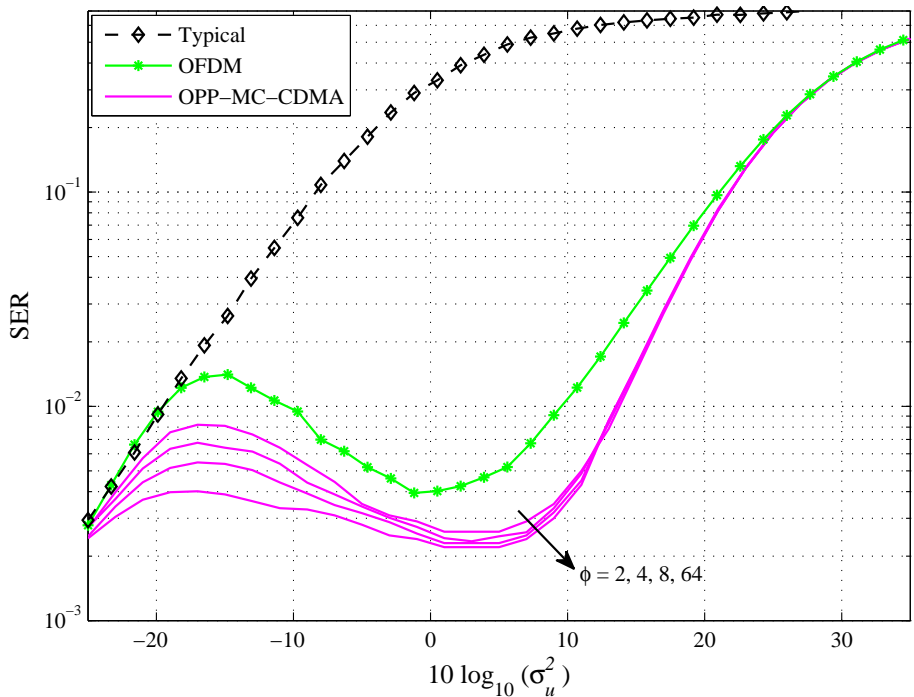

(a) Blanking

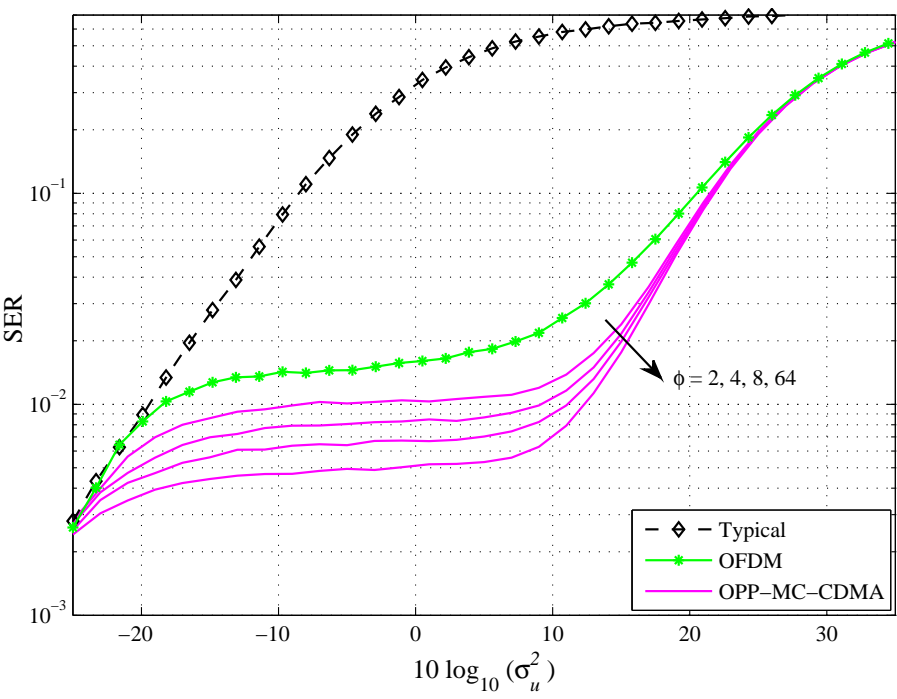

(b) Clipping

Figure 8: Minimum SER performance corresponding to the maximum achievable output SNR versus $10 \log _{10}\left(\sigma_{u}^{2}\right)$ for both MC-CDMA with different values of $\phi$ and OFDM systems in a multipath channel for various codes phases with blanking and clipping. Noise parameters: $A=0.0025$ and $\Gamma=0.001$.

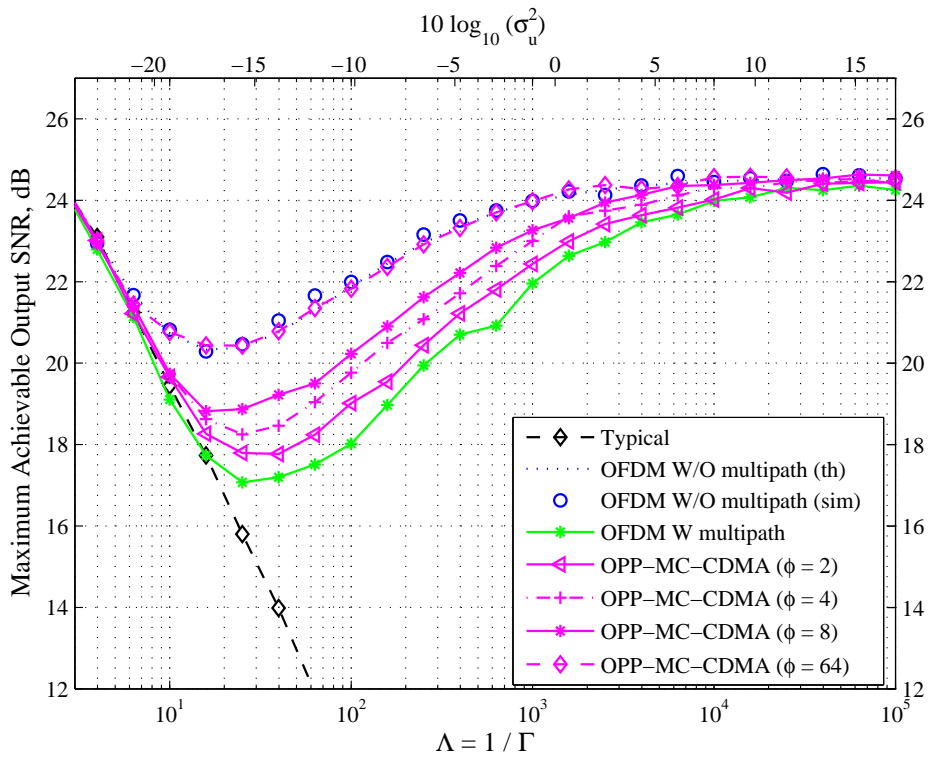

(a) Blanking

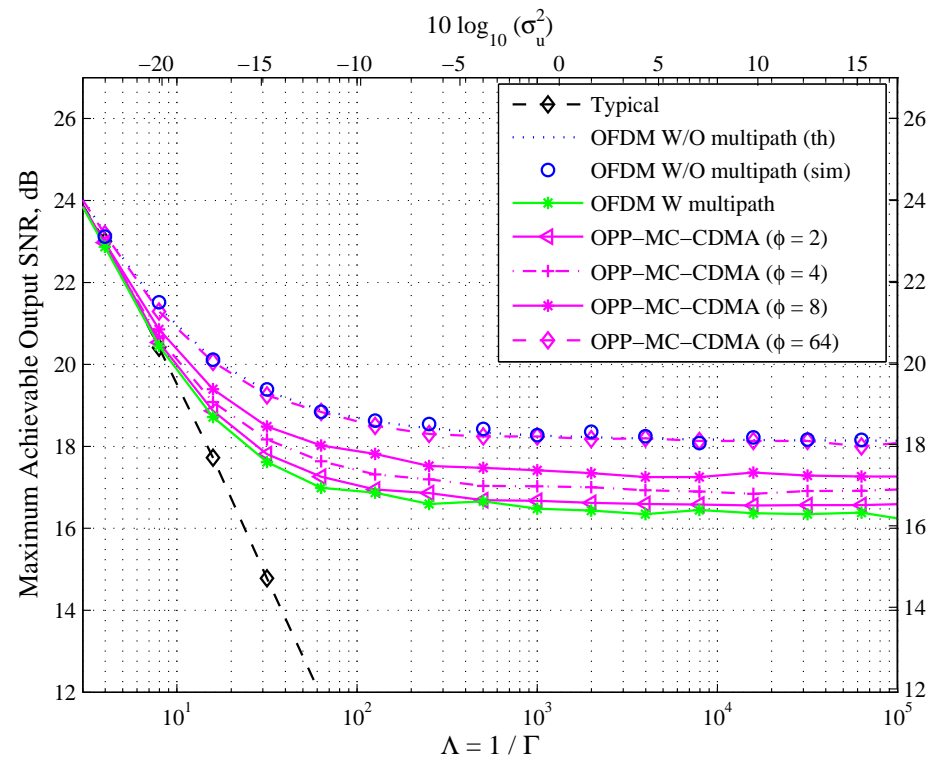

(b) Clipping

Figure 9: Maximum achievable output SNR against $\Lambda$ for both MC-CDMA with various values of $\phi$ and OFDM systems in a multipath channel with blanking and clipping. Noise parameters: $A=0.0025$ and $\mathrm{SGNR}=30 \mathrm{~dB}$.

optimal thresholds become smaller as the number of phases is increased. Although only the optimal thresholds when $\Gamma$ is kept fixed are presented here, below we discuss the corresponding maximum achievable output SNR and minimum achievable SER for two different scenarios depending on whether or not $\Gamma$ is varied.

\section{A. Fixed $\Gamma$}

In this sub-section, we examine the optimized system performance when $\sigma_{u}^{2}$ is varied while fixing $\Gamma$. With this in mind, the maximum achievable output SNR is plotted versus $10 \log _{10}\left(\sigma_{u}^{2}\right)$ as shown in Figs. $7 \mathrm{a}$ and $7 \mathrm{~b}$ for the systems under consideration with blanking and clipping, respectively, when $A=0.0025$ and
$\Gamma=0.001$. Comparing Figs. $7 \mathrm{a}$ and $7 \mathrm{~b}$, few common observations can be seen. Firstly, the typical receiver always has the worst performance followed by the OFDM system with multipath. Secondly, the MC-OPP approach always outperforms the OFDMbased schemes even with a small value of $\phi$ and this enhancement becomes higher as $\phi$ is increased. The third common trend in these figures, and the most interesting, is that the proposed system with $(\phi=64)$ approaches the performance of OFDM W/O multipath over the entire noise spectrum. In general, it is also noticeable that blanking always yields better performance than clipping under same noise and modulation features.

Moreover, it is clear that when $\sigma_{u}^{2}$ is very low, around 25 $\mathrm{dB}$ below transmitted signal level, the performance is very good 


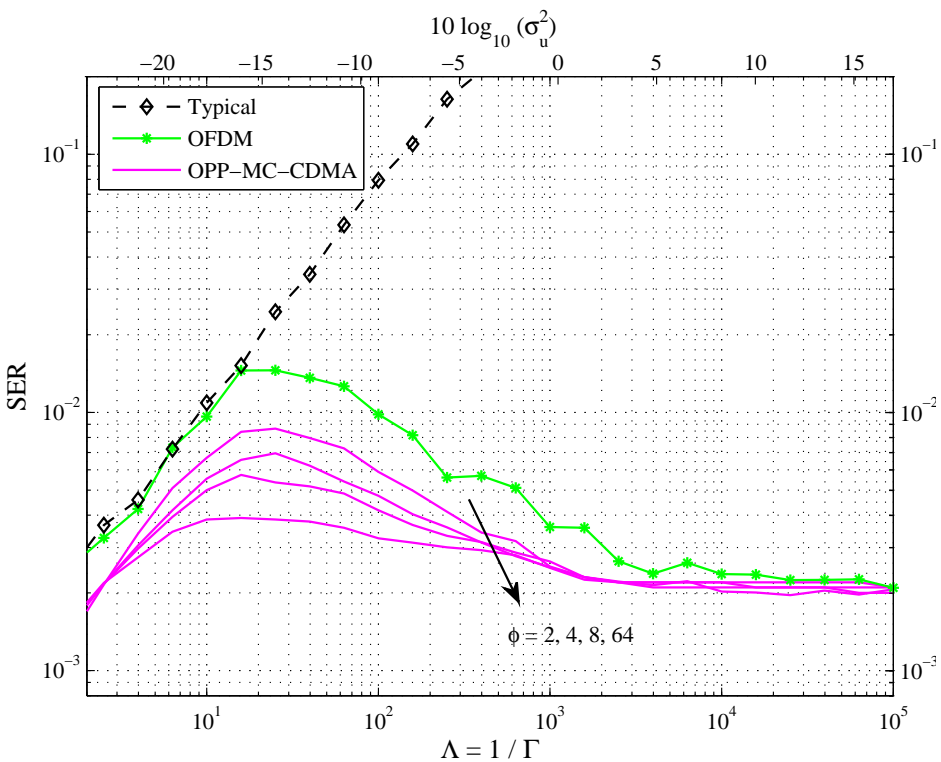

(a) Blanking

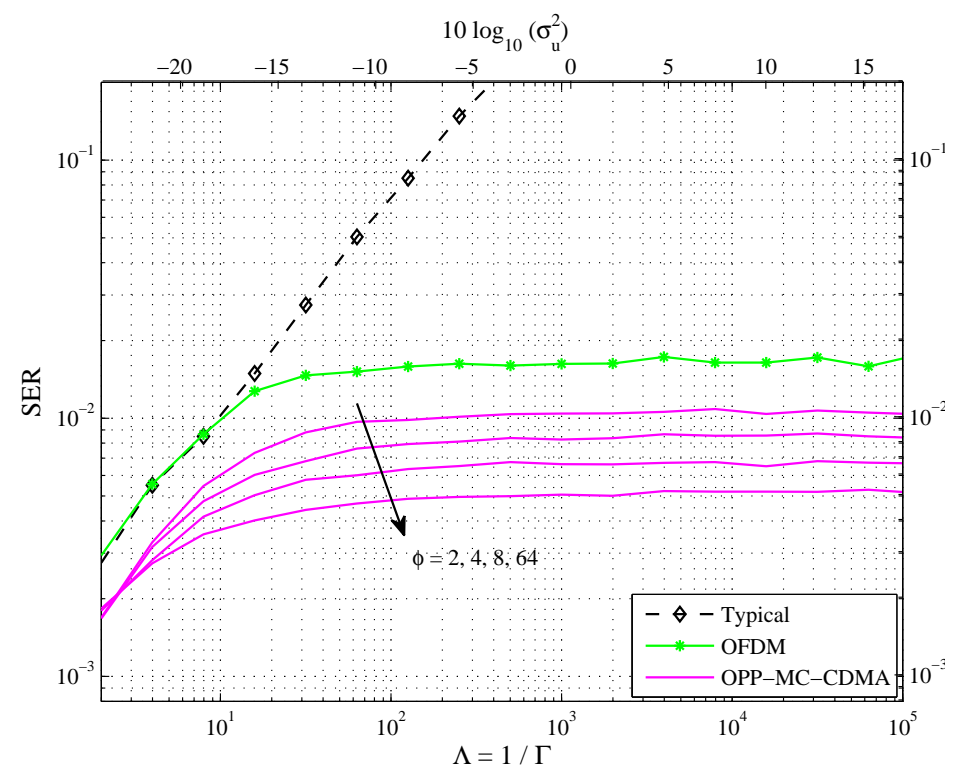

(b) Clipping

Figure 10: Minimum SER performance corresponding to the maximum achievable output SNR against $\Lambda$ for both MC-CDMA with different values of $\phi$ and OFDM systems in a multipath channel with blanking and clipping. Noise parameters: $A=0.0025$ and input SGNR $=30 \mathrm{~dB}$.

for both blanking and clipping which is intuitive. At the other extreme, however, when $\sigma_{u}^{2}$ is very high, around $10 \mathrm{~dB}$ above the signal level, performance deteriorates dramatically. This can be justified by the fact that in this noise region, the system becomes Gaussian limited making the nonlinear preprocessingbased schemes inefficient. However, in the intermediate $\sigma_{u}^{2}$ region $-25 \mathrm{~dB} \lesssim \sigma_{u}^{2} \lesssim 10 \mathrm{~dB}$, it is noted that for the blanking-based systems the output SNRs degrade as $\sigma_{u}^{2}$ increases until it reaches about $-15 \mathrm{~dB}$ after which performance begins to improve reaching its peak at around $\sigma_{u}^{2}=0 \mathrm{~dB}$ with an output SNR of $24 \mathrm{~dB}$ before it declines rapidly again. On the other hand, for the clipping case, it is evident that the output SNR consistently decreases as $\sigma_{u}^{2}$ increases over the entire intermediate region of $\sigma_{u}^{2}$.

For more quantitative characterization of the proposed system, the SER performance in correspondence to the SNRs curves shown in Fig. 7 is found and presented in Fig. 8 for both blanking and clipping from which similar observations can be seen. Notably, blanking has generally lower SER performance but when $\sigma_{u}^{2}$ is extremely high, SER reaches 1 for all considered systems indicating that the nonlinear devices are no longer efficient and, as a consequence, more sophisticated techniques should be utilized instead.

\section{B. Varying $\Gamma$}

In this sub-section we keep $\sigma_{G}^{2}$ fixed such that signal-toGaussian-noise ratio SGNR $=10 \log _{10}\left(\sigma_{x}^{2} / \sigma_{G}^{2}\right)=30 \mathrm{~dB}$ while varying $\sigma_{I}^{2}$. Under these conditions, the output SNRs of the optimized OPP-MC-CDMA and OFDM systems versus $\Lambda=1 / \Gamma$ are illustrated in Fig. 9 with different values of $\phi$. Similar trends as in Fig. 7 can be seen except the fact that when $\Lambda$ is very large, meaning that $\sigma_{u}^{2}$ is also very large as shown on the top $y$ axes, the output SNR does not decline as in the previous subsection. This is owing to the fact that the system is no longer Gaussian limited (since $\sigma_{G}^{2}$ is kept fixed) and IN becomes the dominant parameter in this region. Therefore, when IN is very large it becomes more distinguishable from the useful transmitted signal and, consequently, more efficient banking and clipping process is accomplished. The corresponding SER performance is illustrated in Fig. 10 and similar trends can be noticed.

\section{IMPUlSiVE IndEX IMPACT ON THE OPTIMIZED SySTEMS}

To illustrate the impact of impulsive index on the performance of the proposed system, we set the noise power as $\sigma_{u}^{2}=0.05$ and plot the maximum achievable output SNR of OPP-MC-CDMA with blanking and clipping versus $\phi$ for $A=$ $0.001,0.003,0.005,0.01$, and 0.05 as shown in Fig. 11a. The reason for selecting such noise parameters is to examine a wide range of noisy channels ranging from weakly to heavily disturbed IN environments [14], [39], [40]. It is clearly visible from these results that the output SNR is inversely proportional to $A$ but, as expected, directly proportional to $\phi$. For instance, when $A=0.001$, gains of up to $3 \mathrm{~dB}$ and $2 \mathrm{~dB}$ are attained when $(\phi=64)$ relative to the case when $(\phi=2)$ for the blanking and clipping, respectively; whereas when $A=0.005$ these gains are reduced to around 2.5 $\mathrm{dB}$ and $1.5 \mathrm{~dB}$ for the same features. It is also obvious that the OPP blanking-based scheme performs better than the clipping one when $A$ is low (e.g. $A=0.001,0.003$ and 0.005 ) whereas clipping offers slightly better performance when $A$ is relatively high (e.g. $A=0.01$ and 0.05 ). Furthermore, it is interesting to note that in a heavily-disturbed IN environment $(A=0.05)$ the output SNR becomes almost independent of $\phi$ for both nonlinear preprocessingbased systems. However, it was presented in [9] that such a high IN probability of occurrence is very rare in practice.

For better visualization of the achievable gain over the OFDMbased scheme, we plot the relative gain ( $\left.\mathrm{G}_{\text {OPP-MC-CDMA }}\right)$, which is basically the ratio between the maximum achievable output SNRs of the OPP-MC-CDMA and OFDM systems, and is given by

$$
\mathrm{G}_{\mathrm{OPP}-\mathrm{MC}-\mathrm{CDMA}}=10 \log _{10}\left(\frac{\mathrm{SNR}_{\mathrm{OPP}-\mathrm{MC}-\mathrm{CMDA}}^{(\max )}}{\mathrm{SNR}_{\mathrm{OFDM}}^{(\max )}}\right)
$$




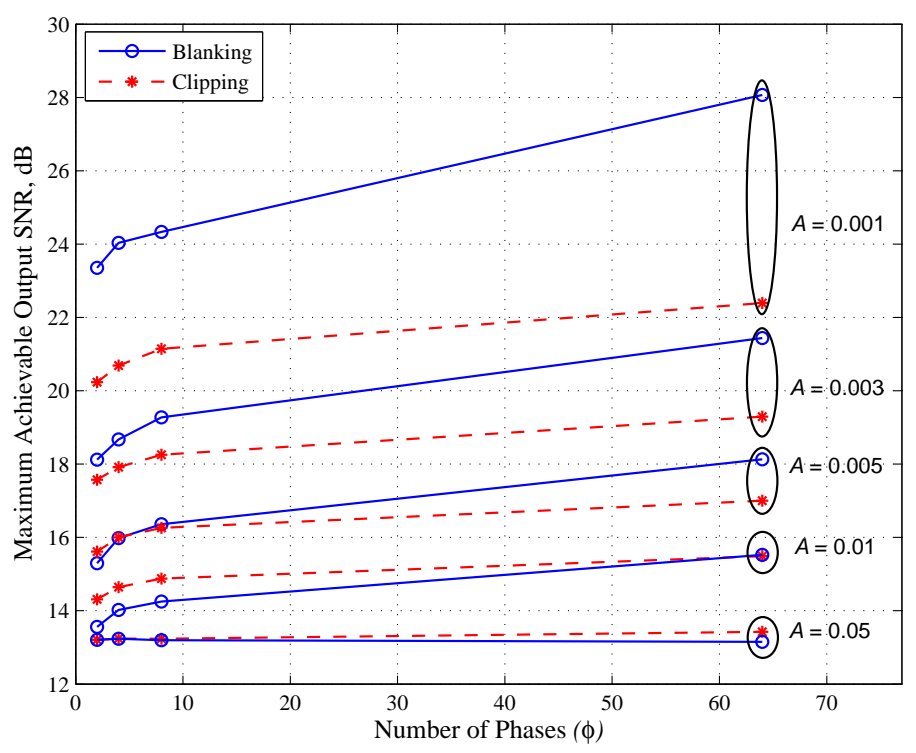

(a) Maximum achievable output SNR

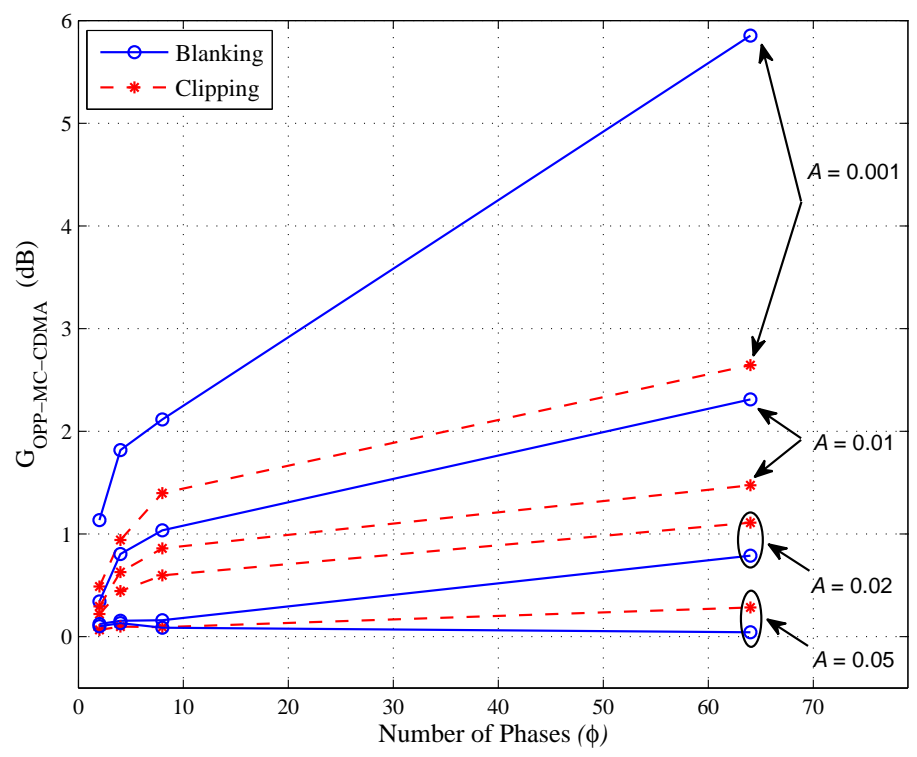

(b) The relative gain

Figure 11: The maximum achievable output SNR and the corresponding relative gain versus the number of OPP codes phases with different values of $A$ for both blanking and clipping in a multipath channel. Noise parameters: $\sigma_{u}=0.05$ and $\Gamma=0.001$.

Fig. $11 \mathrm{~b}$ depicts $\mathrm{G}_{\mathrm{OPP}-\mathrm{MC}-\mathrm{CDMA}}$ versus $\phi$ for several values of $A$ from which it is evident that when $A=0.001$ gains of up to $5.25 \mathrm{~dB}$ and $2.25 \mathrm{~dB}$ can be achieved when $(\phi=64)$ for the blanking and clipping scenarios, respectively. Notably, in a heavilydisturbed IN environment $\mathrm{G}_{\mathrm{OPP}-\mathrm{MC}-\mathrm{CDMA}}$ becomes negligible for clipping and zero for blanking which implies that under such IN conditions OFDM system should be implemented instead since it is less complex. Finally, it must be mentioned at this stage that the improvement the proposed system offers is obtained at the expense of higher complexity of both the transmitter and receiver.

\section{MC-CDMA VERSUS CODED OFDM}

It is well known that the performance of communication systems can be further enhanced with forward error detection techniques. In particular, coding is able to significantly improve the performance of OFDM-based systems in the presence of IN. Numerous codes have been examined over PLC channels such as convolutional codes, low density parity check (LDPC), and Reed-Solomon codes [41]-[45]. Amongst these coding schemes, convolutional codes have been the most widely adopted by various PLC standards [33], [46]-[48]. Therefore, in this section, and for more realistic results, we compare the minimum achievable BER performance of the proposed system to that of coded OFDM. Convolutional codes are used with code rate of $1 / 2$ and constraint length of 7 while the Viterbi algorithm is used for decoding. Simulation parameters adopted here are $N=64$, QPSK modulation, $A=0.1$, input SGNR $=30 \mathrm{~dB}$ and $\phi=64$. Fig. 12 demonstrates the BER performance versus $\Lambda$ for OFDM, coded OFDM and MCCDMA systems with both blanking and clipping at the receiver. For completeness sake, we have also added the BER curves of the typical coded and uncoded OFDM schemes. The first observation one can see is that the proposed OPP-CDMA still always outperforms the other systems for all $\Lambda$ values. It is also apparent, as expected, that coded OFDM improves the BER performance in comparison to the uncoded one. However, it should be highlighted that the performance of the typical coded OFDM approaches the performance of the typical uncoded OFDM when IN is too high, i.e. $\Lambda \gtrsim 10^{3}$. This simply implies that in such IN channels, even coding becomes inefficient and, therefore, other mitigation schemes should be considered such as blanking and/or clipping.

\section{CONCLUSION}

This paper focused on enhancing the reliability of PLC systems for potential implementation in SG applications. To accomplish this, we implemented OPP-based MC-CDMA over frequencyselective power-line channels contaminated with Middleton classA noise. Three burst noise reduction techniques were considered, namely, blanking, clipping and adaptive hybrid. The problem of blanking and clipping threshold optimization was also studied. In comparison to the OFDM-based scheme, it was found that the proposed system is more effective in tackling the PLC channel impairments providing output SNR gains of more than $5 \mathrm{~dB}$ and $2 \mathrm{~dB}$ for the blanking- and clipping-based systems, respectively. It was also demonstrated that the performance of the OPP-MCCDMA approach improves as we increase the constellation size of the OPP codes due to the inverse relationship between the constellation size and the signals' PAPR. Furthermore, for a more reasonable comparison, the BER performance of the coded OFDM, using convolutional codes, was investigated and compared to the proposed system.

\section{REFERENCES}

[1] W. Liu, M. Sigle, and K. Dostert, "Channel characterization and system verification for narrowband power line communication in smart grid applications," IEEE Commun. Mag., vol. 49, no. 12, pp. 28-35, Dec. 2011.

[2] S. Galli, A. Scaglione, and Z. Wang, "For the grid and through the grid: The role of power line communications in the smart grid," Proc. IEEE, vol. 99, no. 6, pp. 998-1027, Jun. 2011.

[3] S. Canale, A. Di Giorgio, A. Lanna, A. Mercurio, M. Panfili, and A. Pietrabissa, "Optimal planning and routing in medium voltage powerline communications networks," IEEE Trans. Smart Grid, vol. 4, no. 2, pp. 711-719, Jun. 2013. 


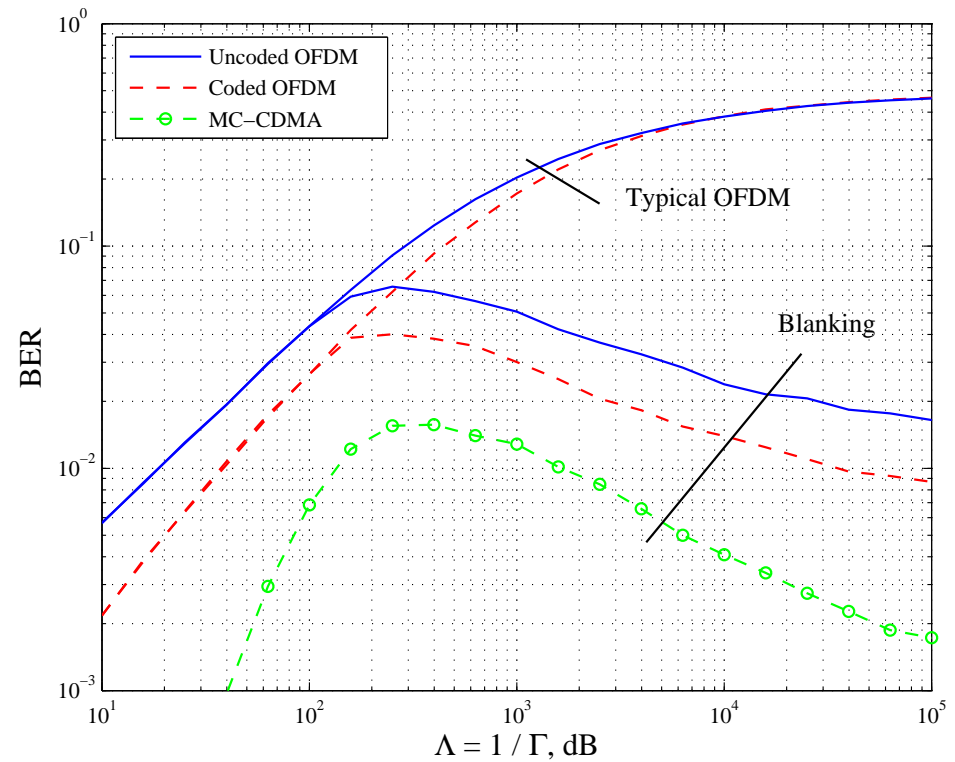

(a) Blanking

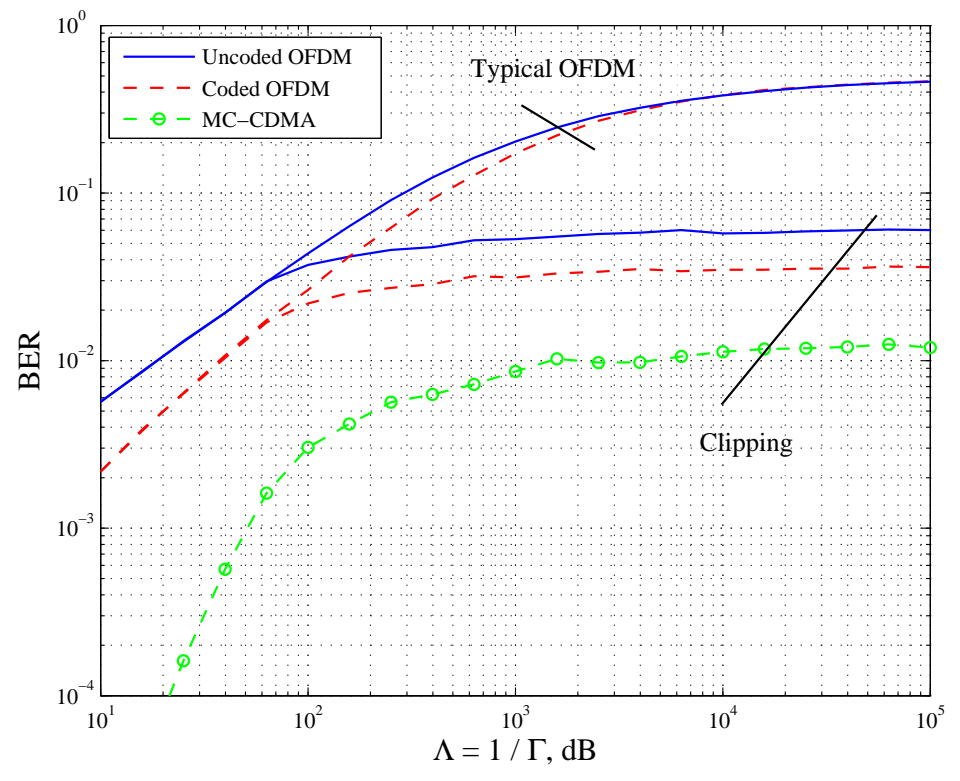

(b) Clipping

Figure 12: Minimum achievable BER performance against $\Lambda$ for the MC-CDMA, uncoded and coded OFDM in a multipath channel with blanking and clipping at the receiver. Noise parameters: $A=0.1$ and input $\mathrm{SGNR}=30 \mathrm{~dB}$.

[4] N. Pavlidou, A. Vinck, J. Yazdani, and B. Honary, "Power line communications: state of the art and future trends," IEEE Commun. Mag., vol. 41, no. 4, pp. 34-40, Apr. 2003.

[5] Q. Liu, B. Zhao, Y. Wang, and J. Hu, "Experience of AMR systems based on BPL in china," in Proc. IEEE Int. Symp. Power Lines Commun. (ISPLC), Mar. 2009, pp. 280-284.

[6] S. Galli, A. Scaglione, and K. Dostert, "Broadband is power: internet access through the power line network," IEEE Commun. Mag., vol. 41, no. 5, pp. 82-83, May 2003.

[7] L. Jianming, Z. Bingzhen, G. Liang, Y. Zhou, and W. Yirong, "Communication performance of broadband PLC technologies for smart grid," in Proc. IEEE Int. Symp. Power Lines Commun. (ISPLC), Apr. 2011, pp. 491-496.

[8] M. Tunc and E. Perrins, "Pilot based channel estimation and transform domain analysis in broadband PLC for smart grid," in Proc. IEEE Int. Conf. Smart Grid Commun. (SmartGridComm), Oct. 2013, pp. 283-288.

[9] M. Zimmermann and K. Dostert, "Analysis and modeling of impulsive noise in broad-band powerline communications," IEEE Trans. Electromagn. Compat., vol. 44, pp. 249-258, Feb. 2002.

[10] M. H. L. Chan and R. Donaldson, "Amplitude, width, and interarrival distributions for noise impulses on intrabuilding power line communication networks," IEEE Trans. Electromagn. Compat., vol. 31, no. 3, pp. 320-323, Aug. 1989.

[11] H. Meng, Y. L. Guan, and S. Chen, "Modeling and analysis of noise effects on broadband power-line communications," IEEE Trans. Power Del., vol. 20 , no. 2, pp. 630-637, Apr. 2005.

[12] M. Gotz, M. Rapp, and K. Dostert, "Power line channel characteristics and their effect on communication system design," IEEE Commun. Mag., vol. 42, no. 4, pp. 78-86, Apr. 2004.

[13] D. Middleton, "Statistical-physical models of urban radio-noise environments - part I: Foundations," IEEE Trans. Electromagn. Compat., vol. EMC-14, no. 2, pp. 38-56, May 1972.

[14] _ "Statistical-physical models of electromagnetic interference," IEEE Trans. Electromagn. Compat., vol. EMC-19, pp. 106-127, Aug. 1977.

[15] J. Matanza, S. Alexandres, and C. Rodriguez-Morcillo, "Difference setsbased compressive sensing as denoising method for narrow-band power line communications," IET Commun., vol. 7, no. 15, pp. 1580-1586, Oct. 2013.

[16] E. Alsusa and K. Rabie, "Dynamic peak-based threshold estimation method for mitigating impulsive noise in power-line communication systems," IEEE Trans. Power Del., vol. 28, no. 4, pp. 2201-2208, Oct. 2013.

[17] S. V. Zhidkov, "Analysis and comparison of several simple impulsive noise mitigation schemes for OFDM receivers," IEEE Trans. Commun., vol. 56, no. 1, pp. 5-9, Jan. 2008.

[18] V. DaSilva and E. Sousa, "Multicarrier orthogonal CDMA signals for quasisynchronous communication systems," IEEE J. Sel. Areas in Commun., vol. 12, no. 5, pp. 842-852, Jun. 1994.

[19] S. Kondo and L. Milstein, "On the use of multicarrier direct sequence spread spectrum systems," in Proc. IEEE Milcom., vol. 1, Oct. 1993, pp. 52-56.
[20] Y. H. Kim, I. Song, S. Yoon, and S. R. Park, "A multicarrier CDMA system with adaptive subchannel allocation for forward links," IEEE Trans. Veh. Technol., vol. 48, no. 5, pp. 1428-1436, Sept. 1999.

[21] "Draft Standard for PoweRline Intelligent Metering Evolution PRIME Alliance Working Group." http://www.prime-alliance.org, accessed: 2014-0825.

[22] "PLC G3 Physical Layer Specification," http://www.erdfdistribution.fr/medias, accessed: 2014-08-25.

[23] S. Mudriievskyi, R. Radeke, and R. Lehnert, "CSMA/CA: Improvements of the contention window adaptation," in Proc. IEEE Int. Symp. Power Lines Commun. (ISPLC), Mar. 2013, pp. 53-57.

[24] L. Di Bert, S. D'Alessandro, and A. Tonello, "MAC enhancements for G3PLC home networks," in Proc. IEEE Int. Symp. Power Lines Commun. (ISPLC), Mar. 2013, pp. 155-160.

[25] M. Crussiere, J. Baudais, and J. Helard, "Adaptive spread-spectrum multicarrier multiple-access over wirelines," IEEE J. Sel. Areas in Commun., vol. 24, no. 7, pp. 1377-1388, Jul. 2006.

[26] M. Crussiere, J. Baudais, and J. F. Helard, "Improved throughput over wirelines with adaptive MC-DS-CDMA," in Proc. IEEE Int. Symp. Spread Spectr. Techn. Appl., Aug. 2006, pp. 143-147.

[27] J.-Y. B. et M. CrussiÃ̂́lre, "Resource allocation with adaptive spread spectrum OFDM using 2D spreading for power line communications," EURASIP $J$. Advances Signal Process., vol. 2007, pp. 1-13, 2007.

[28] S. Hara and R. Prasad, "Overview of multicarrier CDMA," IEEE Commun. Mag., vol. 35, no. 12, pp. 126-133, Dec. 1997.

[29] A. Viterbi, "Very low rate convolution codes for maximum theoretical performance of spread-spectrum multiple-access channels," IEEE J. Sel. Areas in Commun., vol. 8, no. 4, pp. 641-649, May 1990.

[30] R. Kirlin, "Pseudo-random analog noise generation," Proc. IEEE, vol. 64, no. 5, pp. 824-826, May 1976.

[31] K. Rabie and E. Alsusa, "Preprocessing based impulsive noise reduction for power-line communications," IEEE Trans. Power Del., vol. 29, no. 4, pp. 1648-1658, Aug. 2014.

[32] J. Tellado, Multicarrier Modulation With Low PAR: Applications to DSL and Wireless. Norwell, MA: Kluwer, 2000.

[33] M. Nassar, J. Lin, Y. Mortazavi, A. Dabak, I. H. Kim, and B. Evans, "Local utility power line communications in the 3-500 khz band: Channel impairments, noise, and standards," IEEE Signal Process. Mag., vol. 29, no. 5, pp. 116-127, Sept. 2012.

[34] M. Katayama, T. Yamazato, and H. Okada, "A mathematical model of noise in narrowband power line communication systems," IEEE J. Sel. Areas in Commun., vol. 24, no. 7, pp. 1267-1276, Jul. 2006.

[35] M. Nassar, A. Dabak, I. H. Kim, T. Pande, and B. Evans, "Cyclostationary noise modeling in narrowband powerline communication for smart grid applications," in Proc. IEEE Int. Conf. Acoustics, Speech and Signal Process. (ICASSP), Mar. 2012, pp. 3089-3092. 
[36] K. Rabie and E. Alsusa, "Threshold and scaling factor optimization for

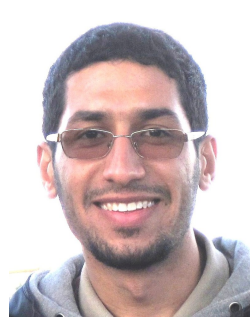

Khaled Maaiuf Rabie (S'12), received the B.Sc. degree (with Hons.) in Electrical and Electronic Engineering from the University of Tripoli, Tripoli, Libya, in 2008 and the M.Sc. degree (with Hons.) in Communication Engineering from the University of Manchester, Manchester, UK, in 2010, where he is currently pursuing the Ph.D. degree in Microwave and Communications Systems (MCS). His current research interests are power-line communications, including power-line channel modeling, interference, and impulsive noise mitigation for smart-grid applications as well as multi-input multi-output systems. He received the best student paper award at the IEEE International Symposium on Power Line Communications (ISPLC) in 2015.

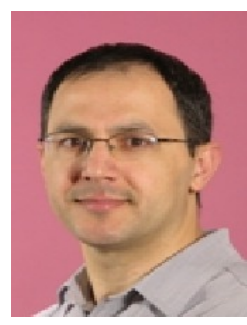

Emad Alsusa (M'06-SM'07) received a Ph.D. degree in electrical and electronic engineering from Bath University, Bath in 2000. Following his PhD he joined the School of Engineering and Electronics at Edinburgh University as a MobileVCE Postdoctoral Research Fellow, working on industrially led projects on link enhancement techniques for future high data rate wireless communication systems. In 2003, he joined the University of Manchester as an academic member of the school of Electrical and Electronic Engineering, where he lectures on communication engineering subjects. His research interests include signal processing and analysis of wireless communication networks, with particular focus on modulation and multiple access, channel estimation, coding, interference mitigation, multiuser detection, MIMO techniques and spectrum sensing techniques for cognitive radio applications. He served as a technical program committee member on numerous IEEE flagship conferences and chaired the Manchester EEE postgraduate conference in 2010 . enhancing impulsive noise cancellation in PLC systems," in Proc. IEEE Global Commun. Conf. (GLOBECOM), Dec. 2014, pp. 2977-2982.

[37] A. Goldsmith, Wireless Communications. Cambridge University Press, 2005.

[38] V. Tarokh and H. Jafarkhani, "On the computation and reduction of the peak-to-average power ratio in multicarrier communications," IEEE Trans. commun., vol. 48, no. 1, pp. 37-44, Jan. 2000.

[39] S. Lai and G. Messier, "Using the wireless and PLC channels for diversity," IEEE Trans. Commun, vol. 60, no. 12, pp. 3865-3875, Dec. 2012.

[40] B. Nikfar, T. Akbudak, and A. Vinck, "MIMO capacity of class A impulsive noise channel for different levels of information availability at transmitter," in Proc. IEEE Int. Symp. Power Lines Commun. (ISPLC), Mar. 2014, pp. 266-271.

[41] D. Umehara, H. Yamaguchi, and Y. Morihiro, "Turbo decoding in impulsive noise environment," in Proc. IEEE Global Telecommun. Conf. (GLOBECOM), vol. 1, Nov. 2004, pp. 194-198.

[42] N. Andreadou and F.-N. Pavlidou, "PLC channel: Impulsive noise modelling and its performance evaluation under different array coding schemes," IEEE Trans. Power Del., vol. 24, no. 2, pp. 585-595, Apr. 2009.

[43] H.-M. Oh, Y.-J. Park, S. Choi, J.-J. Lee, and K.-C. Whang, "Mitigation of performance degradation by impulsive noise in LDPC coded OFDM system," in Proc. IEEE Int. Symp. Power Lines Commun. (ISPLC), 2006, pp. 331-336.

[44] H. Nguyen and T. Bui, "Bit-interleaved coded OFDM with iterative decoding in impulsive noise," IEEE Trans. Power Del., vol. 23, no. 2, pp. 640-649, Apr. 2008.

[45] N. Andreadou and F.-N. Pavlidou, "Mitigation of impulsive noise effect on the PLC channel with QC-LDPC codes as the outer coding scheme," IEEE Trans. Power Del., vol. 25, no. 3, pp. 1440-1449, Jul. 2010.

[46] I. Kim, B. Varadarajan, and A. Dabak, "Performance analysis and enhancements of narrowband OFDM powerline communication systems," in Proc. IEEE Int. Conf. Smart Grid Commun. (SmartGridComm), Oct. 2010, pp. 362 367.

[47] HomePlug AV White Paper. HomePlug Powerline Alliance, 2005. 